\title{
Adaptation of prey and predators between patches
}

\author{
Wendi Wang ${ }^{\dagger}, a$, Yasuhiro Takeuchi* \\ $\dagger$ : Key Laboratory of Eco-environments in Three Gorges Reservoir Region, \\ School of Mathematics and Statistics, Southwest University, \\ Chongqing, 400715, P. R. China. \\ E-mail: wendi_wangswu@yahoo.com.cn \\ *: Graduate School of Science and Technology, Shizuoka University, \\ Hamamatsu 432-8561, Japan. \\ E-mail: takeuchi@sys.eng.shizuoka.ac.jp
}

$a$ : The research was supported by National Science Fund of China (10871162) and HeiwaNakajima Fund. 
2 Mathematical models are proposed to simulate migrations of prey and predators 3 between patches. In the absence of predators, it is shown that the adaptation of ${ }_{4}$ prey leads to an ideal spatial distribution in the sense that the maximal capacity 5 of each patch is achieved. With the introduction of co-adaptation of predators, 6 it is proved that both prey and predators achieve ideal spatial distributions when 7 the adaptations are weak. Further, it is shown that the adaptation of prey and 8 predators increases the survival probability of predators from the extinction in both 9 patches to the persistence in one patch. It is also demonstrated that there exists 10 a pattern that prey and predators cooperate well through adaptations such that 11 predators are permanent in every patch in the case that predators become extinct 12 in each patch in the absence of adaptations. For strong adaptations, it is proved 13 that the model admits periodic cycles and multiple stability transitions. Key Words: Migration; distribution; stability; persistence; cycle. 


\section{${ }_{15} \mathbf{1}$ Introduction}

Population dispersal is one of the most important subjects in ecology. It is well recognized that the spatial distribution of populations and population dynamics are much affected by spatial heterogeneity and population mobility (Shigesada and Kawasaki, 1997; Namba, Umemoto, and Minami, 1999; Diekmann, Law, and Meta, 2000; Murray, 2003; Arino and van den Driessche, 2006; Takeuchi, Iwasa, and Sato, 2007; Lou, 2008). For fragmented landscapes, which are common because populations of most species occupy mosaic habitats and because of rapid destruction of natural habitats (Watkinson and Sutherland, 1995; Hanski, 1998), patch models are ideal tools to mimic population dynamics. Within each patch, individuals in each population are assumed to be identical and can migrate to other patches. Levin (1974) proposed the two-species competition and prey-predator models with population dispersal among patches. Lu and Takeuchi (1993) found that population dispersals of single species among patches enhance the stability of populations. For interactions of predators and prey, Takeuchi (1986) and Kuang and Takeuchi (1994) found that stabilizing and destabilizing effects could be induced by prey dispersal. More recently, Briggs and Hoopes (2004) identify three mechanisms whereby limited dispersal of hosts and parasitoids combined with other features, such as spatial and temporal heterogeneity, can promote persistence and stability of populations.

The common points for most of papers in the studies of interactions of prey and predators with patchy structures are the assumptions of density independent dispersal (constant traits of species) or random mobility (spatial flows of populations move towards the patch with lower density and are proportional to their density differences between patches). This means that population migration rates are not influenced by biotic conditions. However, many observations indicate that popula- 
tion dispersal rates may be regulated by population densities and habitat qualities. Indeed, the density-dependent dispersal rates of populations were experimentally observed in mites (Bernstein, 1984), insects (Fonseca and Hart, 1996) and vertebrates (French and Travis, 2001; Matthysen, 2005). More recently, by using aquatic experimental microcosms under controlled conditions, Hauzy et al. (2007) observed that the dispersal rate of prey is influenced by its own density and predator's density, and the dispersal rate of predators is affected by prey's density. As a matter of fact, prey migrations may be accelerated by poor reproduction conditions, high predation risks in local habitat, or because of attraction from better reproductions or less predation pressure at other patches, and predators may change behaviors on the basis of prey abundance and demographic advantages (Abrams, 2007; Abrams et al., 2007; Cressman and Krivan, 2006; DeAngelis et al., 2006; Hastings, 1983; Hofbauer and Sigmund, 1998; Kisdi and Liu, 2006; Persson and de Roos, 2003). To understand the effects of density-dependent dispersal of populations, Chiorino et al. (1999); Mchich et al. (2007) and Abdllaoui et al. (2007) considered predator-prey models in a two-patch environment, where prey leave a patch at a migration rate proportional to the local predator density and predators leave a patch at a migration rate inversely proportional to local prey population density. This means that prey's emigration is determined by predation pressure and predator's migration is mainly affected by prey's abundance. Based upon the assumption that the time scale of population migrations between patches is much faster than that of prey growth, predator mortality and predator-prey interactions, they find that for a large class of density-dependent migration rules for predators and prey there exists a unique and stable equilibrium for migration. Moreover, under some particular conditions, the density dependence of migrations can generate a limit cycle. de Roos et al. (2002) and Persson and de Roos (2003) examine flexible behaviors in size-structured populations by assuming that the movement rate out 
of a patch is purely a function of fitness of individuals within that patch. Note that individuals may assess benefits and costs of migrations by learning to decide whether to leave current patch or not. For prey individuals, they may compare the difference of predation pressure between the home patch and a destination patch. For predator individuals, they could compute the difference of food richness of the two patches. Furthermore, the risk of higher mortality of the destination habitat should also be considered. To explore the role of such habitat choice behaviors, let us consider two patches, labeled by 1 and 2 , and $W_{i}$ be the instantaneous per capita growth rate for an individual in patch $i$. Then the per individual dispersal rate from patch $i$ to patch $j$ is assumed (Abrams, 2000, 2003; Abrams and Matsuda, 2004; Abrams, 2007; Abrams et al., 2007) to be given by $m \exp \left(\lambda\left(W_{j}-W_{i}\right)\right)$, where $m$ is the basal per capita movement rate from patch $i$ to patch $j$ when fitness is equal and $\lambda$ is a positive constant that represents the sensitivity of movement to a fitness difference. In contrast with the models that separate behavioral dynamics and population dynamics, the combined behavioral-population systems indicate that spatial cycling can stabilize population densities and qualitatively change the responses of population densities to environmental perturbations (Abrams, 2007). Note that the movement rule in previous studies is among possible choices and different movement rules can cause significant quantitative differences in population dynamics (Abrams, 2007).

In this paper, we hope to extend the research by introducing two dynamical variables to represent the adaptations of dispersal rates of prey and predators. This alternative approach gives an advantage that more mathematical analysis can be carried out. Especially, we can show that optimal states can be achieved by prey and predators through adaptations and the adaptations can enhance the survival probability of populations. Therefore, biological insights can be provided in a different way. 
The organization of this paper is as follows. In the next section, we present the formulation of mathematical model. Section 3 shows that ideal spatial distributions of populations can be established through weak adaptations. In section 4 , we show that the adaptations can increase the permanence of populations. Finally, we show that large adaptations induce complicated dynamical behaviors.

\section{Model formulations}

We consider two patches. Let $x_{i}$ be the density of prey in patch $i$ and $y_{i}$ be the density of predators in patch $i$. Without population dispersal, we assume that the interaction of prey and predators is described by

$$
\left\{\begin{array}{l}
\frac{d x_{i}}{d t}=x_{i} g_{i}\left(x_{i}\right)-f_{i}\left(x_{i}\right) y_{i}, \\
\frac{d y_{i}}{d t}=y_{i}\left(k_{i} f_{i}\left(x_{i}\right)-d_{i}\right),
\end{array}\right.
$$

where $g_{i}$ is the per capita growth rate of the prey in patch $i, d_{i}$ is the per capita death rate of predators in patch $i, f_{i}$ is the functional response of predators in patch $i$ and $k_{i}$ is the conversion coefficient.

In order to incorporate population movements between the two patches, we consider those individuals of prey or predators that have ability and desire to disperse in unit time, which are called movable individuals. Movable individuals decide their habitats after comparing costs and benefits of migrations. Let $u$ denote the ratio of movable individuals in prey to current individuals in prey in unit time, and $v$ denote the ratio of movable individuals in predators to current individuals in predators in unit time. If $m_{1}, 0 \leq m_{1} \leq 1$, is the migration probability of a movable individual of prey from the first patch to the second patch, we assume that the migration probability for a movable individual of prey to migrate from the second patch to the first patch is complementary to the probability of a movable 
individual of prey from the first patch to the second patch, i.e., $1-m_{1}$. Indeed, the migration probability $m_{1}$ just means that a prey individual in the first patch has the desirability $m_{1}$ to stay in the second patch and the desirability $1-m_{1}$ to stay in the first patch, after evaluating the qualities of two patches. Since we assume that each individual knows clearly the conditions of two patches, all members of prey have the same desire to stay in a fixed patch wherever they stay. Hence, a prey individual in the second patch has also the desirability $m_{1}$ to stay in the second patch and the desirability $1-m_{1}$ to stay in the first patch. As a consequence, $1-m_{1}$ is the migration probability of a movable individual of prey from the second patch to the first patch. For example, if the quality of patch 2 is 2 times better than that of patch 1 , then the probability that a prey individual has the desire to stay in the first patch is $1 / 3$ and the probability to stay in the second patch is $2 / 3$. Hence, the migration probability from the first patch to the second patch is $m_{1}=2 / 3$ and the migration probability from the second patch to the first patch is $1-m_{1}=1 / 3$. Similarly, we let $n_{1}$ denote the migration probability of a movable predator to migrate from the first patch to the second patch and $1-n_{1}$ denote the probability of a movable predator to disperse from the second patch to the first patch. With the inclusion of population dispersal (2.1) is modified into:

$$
\left\{\begin{array}{l}
\frac{d x_{1}}{d t}=x_{1}\left(g_{1}\left(x_{1}\right)-u m_{1}\right)-f_{1}\left(x_{1}\right) y_{1}+u\left(1-m_{1}\right) x_{2}, \\
\frac{d y_{1}}{d t}=y_{1}\left(k_{1} f_{1}\left(x_{1}\right)-d_{1}-v n_{1}\right)+v\left(1-n_{1}\right) y_{2}, \\
\frac{d x_{2}}{d t}=x_{2}\left(g_{2}\left(x_{2}\right)-u\left(1-m_{1}\right)\right)-f_{2}\left(x_{2}\right) y_{2}+u m_{1} x_{1}, \\
\frac{d y_{2}}{d t}=y_{2}\left(k_{2} f_{2}\left(x_{2}\right)-d_{2}-v\left(1-n_{1}\right)\right)+v n_{1} y_{1} .
\end{array}\right.
$$

To include the adaptations of migration probabilities $m_{1}$ and $n_{1}$ in the process of population fluctuations, we assume that the migration process of prey and predators is so quick that their fitness is determined by the within-habitat population 
dynamics. Note that the fitness of prey in the first patch is

$$
g_{1}\left(x_{1}\right)-f_{1}\left(x_{1}\right) y_{1} / x_{1}
$$

and the fitness of prey in the second patch is

$$
g_{2}\left(x_{2}\right)-f_{2}\left(x_{2}\right) y_{2} / x_{2}
$$

If an individual of prey in the first patch takes migration probability $m_{1}$, then its expected fitness is

$$
E=m_{1}\left(g_{2}\left(x_{2}\right)-f_{2}\left(x_{2}\right) y_{2} / x_{2}\right)+\left(1-m_{1}\right)\left(g_{1}\left(x_{1}\right)-f_{1}\left(x_{1}\right) y_{1} / x_{1}\right),
$$

because its probability to stay in the second patch is $m_{1}$ and the probability to stay in the first patch is $1-m_{1}$. Let $D$ denote the difference between the fitness to stay in the second patch and the expected fitness $E$ :

$$
\begin{aligned}
D & =\left(g_{2}\left(x_{2}\right)-f_{2}\left(x_{2}\right) y_{2} / x_{2}\right)-E \\
& =\left(1-m_{1}\right)\left(g_{2}\left(x_{2}\right)-f_{2}\left(x_{2}\right) y_{2} / x_{2}-\left(g_{1}\left(x_{1}\right)-f_{1}\left(x_{1}\right) y_{1} / x_{1}\right)\right) .
\end{aligned}
$$

Following the principle of replication dynamics (Hofbauer and Sigmund, 1998), which is the cornerstone of evolutionary dynamics, we suppose that the rate of increase of $\frac{d m_{1}}{d t} / m_{1}$ is proportional to the fitness difference $D$ to obtain

$$
\frac{d m_{1}}{d t}=g m_{1}\left(1-m_{1}\right)\left\{\left[g_{2}\left(x_{2}\right)-\frac{f_{2}\left(x_{2}\right) y_{2}}{x_{2}}\right]-\left[g_{1}\left(x_{1}\right)-\frac{f_{1}\left(x_{1}\right) y_{1}}{x_{1}}\right]\right\}
$$

where $g$ is the proportional constant. It is easy to see that (2.5) implies that $0<m_{1}(t)<1$ for $t>0$ if $0<m_{1}(0)<1$. Note that the expression in the first square brackets of (2.5) is the fitness of prey in the second patch and the expression 
in the second square brackets of (2.5) is the fitness of prey in the first patch. It follows from (2.5) that the migration probability $m_{1}$ of prey from the first patch to the second patch increases if the second patch is better than the first patch for prey, decreases if the first patch is better than the second patch for prey, and keeps constant if the two patches have the same quality.

Similarly, we can present the dynamical equation for $n_{1}$ :

$$
\frac{d n_{1}}{d t}=h n_{1}\left(1-n_{1}\right)\left(k_{2} f_{2}\left(x_{2}\right)-d_{2}-\left(k_{1} f_{1}\left(x_{1}\right)-d_{1}\right)\right)
$$

where $h$ is the proportional constant. Consequently, the full model with population dynamics and adaption dynamics is given by

$$
\left\{\begin{aligned}
\frac{d x_{1}}{d t} & =x_{1}\left(g_{1}\left(x_{1}\right)-u m_{1}\right)-f_{1}\left(x_{1}\right) y_{1}+u\left(1-m_{1}\right) x_{2} \\
\frac{d y_{1}}{d t} & =y_{1}\left(k_{1} f_{1}\left(x_{1}\right)-d_{1}-v n_{1}\right)+v\left(1-n_{1}\right) y_{2} \\
\frac{d x_{2}}{d t} & =x_{2}\left(g_{2}\left(x_{2}\right)-u\left(1-m_{1}\right)\right)-f_{2}\left(x_{2}\right) y_{2}+u m_{1} x_{1} \\
\frac{d y_{2}}{d t} & =y_{2}\left(k_{2} f_{2}\left(x_{2}\right)-d_{2}-v\left(1-n_{1}\right)\right)+v n_{1} y_{1}, \\
\frac{d m_{1}}{d t} & =g m_{1}\left(1-m_{1}\right)\left(g_{2}\left(x_{2}\right)-\frac{f_{2}\left(x_{2}\right) y_{2}}{x_{2}}-\left(g_{1}\left(x_{1}\right)-\frac{f_{1}\left(x_{1}\right) y_{1}}{x_{1}}\right)\right) \\
\frac{d n_{1}}{d t} & =h n_{1}\left(1-n_{1}\right)\left(k_{2} f_{2}\left(x_{2}\right)-d_{2}-\left(k_{1} f_{1}\left(x_{1}\right)-d_{1}\right)\right) .
\end{aligned}\right.
$$

We assume that $\lim _{x_{i} \rightarrow 0} f_{i}\left(x_{i}\right) / x_{i}=\alpha_{i} \geq 0$. Then (2.7) is well defined for $x_{i} \geq$ $0, y_{i} \geq 0$ with $i=1,2,0 \leq m_{1} \leq 1$ and $0 \leq n_{1} \leq 1$

\section{Ideal distribution through adaptations}

In this section, we show that populations can achieve ideal spatial distributions through weak adaptations. We begin from the special case that predators are 
absent. Without predators (2.7) is reduced to

$$
\left\{\begin{aligned}
\frac{d x_{1}}{d t} & =x_{1}\left(g_{1}\left(x_{1}\right)-u m_{1}\right)+u\left(1-m_{1}\right) x_{2} \\
\frac{d x_{2}}{d t} & =x_{2}\left(g_{2}\left(x_{2}\right)-u\left(1-m_{1}\right)\right)+u m_{1} x_{1} \\
\frac{d m_{1}}{d t} & =g m_{1}\left(1-m_{1}\right)\left(g_{2}\left(x_{2}\right)-g_{1}\left(x_{1}\right)\right) .
\end{aligned}\right.
$$

To ensure the survival of prey in the absence of predators, we make an assumption

(H1) There exists a $C_{i}$ such that $g_{i}\left(C_{i}\right)=0, g_{i}\left(x_{i}\right)>0$ for $0 \leq x_{i}<C_{i}$ and $g_{i}\left(x_{i}\right)<0$ for $x_{i}>C_{i}$.

Then $C_{i}$ is the carrying capacity of prey in patch $i$. We say that ideal states of prey are achieved if its carrying capacity in every patch is established.

Theorem 1 Let (H1) hold. Then system (3.1) admits a unique positive equilibrium $E_{\text {prey }}=\left(C_{1}, C_{2}, C_{2} /\left(C_{1}+C_{2}\right)\right)$.

The next theorem states that the unique positive equilibrium is globally stable if the adaptation is weak (proofs of theorem 1 and theorem 2 are postponed to Appendix).

Theorem 2 Let $(H 1)$ hold and $g_{i}^{\prime}\left(x_{i}\right)<0$. Then $E_{\text {prey }}$ is globally stable if $0<g \ll$ 1.

Theorem 2 indicates that the carrying capacity of prey population in each patch is achieved through weak adaptations. Hence, an ideal spatial distribution is established. Notice that $E_{\text {prey }}$ is asymptotically stable irrespective of magnitude of adaptation coefficient $g$. For the growth rates of logistic type, extensive numerical calculations indicate that the equilibrium $E_{\text {prey }}$ is also globally stable for large $g$. This suggests that the adaptation always facilitates the formation of ideal spatial distribution of prey. 
Now, a natural question is what will happen when predators are present. By using the same arguments as above, we obtain

Theorem 3 Let (H1) hold. Suppose that functional responses $f_{i}$ are strictly increasing functions and

$$
f_{i}\left(C_{i}\right)>\frac{d_{i}}{k_{i}}, \quad i=1,2 .
$$

Then system (2.7) admits a unique positive equilibrium $E^{*}=\left(x_{1}^{*}, y_{1}^{*}, x_{2}^{*}, y_{2}^{*}, m_{1}^{*}, n_{1}^{*}\right)$, where

$$
\begin{array}{lll}
x_{i}^{*}=f_{i}^{-1}\left(\frac{d_{i}}{k_{i}}\right), & y_{i}^{*}=\frac{x_{i}^{*} g_{i}\left(x_{i}^{*}\right)}{\frac{d_{i}}{k_{i}}}, & i=1,2, \\
m_{1}^{*}=\frac{x_{2}^{*}}{x_{1}^{*}+x_{2}^{*}}, & n_{1}^{*}=\frac{y_{2}^{*}}{y_{1}^{*}+y_{2}^{*}}, & i=1,2 .
\end{array}
$$

Note that $\left(x_{i}^{*}, y_{i}^{*}\right)$ with components defined in (3.3) is the coexistence state of prey and predators in the isolated patch $i$. If this state is globally asymptotically stable in the isolated patch $i$, then the community of prey population and predator population evolves to it through natural selection. Similar to the case of single species, we call it as the carrying capacity of the ecological community of the prey population and the predator population in patch $i$.

Note that $m_{1}^{*}\left(n_{1}^{*}\right)$ given in (3.3) implies that the migration rate of prey (predators) to patch $1 u\left(1-m_{1}^{*}\right) x_{2}^{*}\left(v\left(1-n_{1}^{*}\right) y_{2}^{*}\right)$ equals the emigration rate of prey (predators) from patch $1 u m_{1}^{*} x_{1}^{*}\left(v n_{1}^{*} y_{1}^{*}\right)$. We expect that positive solutions of $(2.7)$ approach $E^{*}$ as $t \rightarrow \infty$ for small $g$ and $h$, which means that prey population and predator population in patch $i$ evolve, under weak adaptations of both prey and predators, to carrying capacity of the ecological community. To be tractable in analysis, we suppose that prey grow according to the logistic law:

$$
g_{i}\left(x_{i}\right)=r_{i}-\mu_{i} x_{i}, \quad i=1,2
$$


and the functional responses are linear $f_{i}\left(x_{i}\right)=b_{i} x_{i}$. Then (2.7) is reduced to:

$$
\left\{\begin{aligned}
\frac{d x_{1}}{d t} & =x_{1}\left(r_{1}-\mu_{1} x_{1}-b_{1} y_{1}\right)-u m_{1} x_{1}+u\left(1-m_{1}\right) x_{2}, \\
\frac{d y_{1}}{d t} & =y_{1}\left(k_{1} b_{1} x_{1}-d_{1}-v n_{1}\right)+v\left(1-n_{1}\right) y_{2}, \\
\frac{d x_{2}}{d t} & =x_{2}\left(r_{2}-\mu_{2} x_{2}-b_{2} y_{2}\right)-u\left(1-m_{1}\right) x_{2}+u m_{1} x_{1}, \\
\frac{d y_{2}}{d t} & =y_{2}\left(k_{2} b_{2} x_{2}-d_{2}-v\left(1-n_{1}\right)\right)+v n_{1} y_{1}, \\
\frac{d m_{1}}{d t} & =g m_{1}\left(1-m_{1}\right)\left(r_{2}-\mu_{2} x_{2}-b_{2} y_{2}-\left(r_{1}-\mu_{1} x_{1}-b_{1} y_{1}\right)\right) \\
\frac{d n_{1}}{d t} & =h n_{1}\left(1-n_{1}\right)\left(k_{2} b_{2} x_{2}-d_{2}-\left(k_{1} b_{1} x_{1}-d_{1}\right)\right) .
\end{aligned}\right.
$$

By Theorem 3, (3.4) has a unique positive equilibrium $E^{*}=\left(x_{1}^{*}, y_{1}^{*}, x_{2}^{*}, y_{2}^{*}, m_{1}^{*}, n_{1}^{*}\right)$ if

$$
r_{i} k_{i} b_{i}>d_{i} \mu_{i}, \quad i=1,2
$$

where

$$
\begin{aligned}
& x_{1}^{*}=\frac{d_{1}}{k_{1} b_{1}}, \quad x_{2}^{*}=\frac{d_{2}}{k_{2} b_{2}}, \quad y_{1}^{*}=\frac{r_{1} k_{1} b_{1}-\mu_{1} d_{1}}{k_{1} b_{1}^{2}}, \\
& y_{2}^{*}=\frac{r_{2} k_{2} b_{2}-\mu_{2} d_{2}}{k_{2} b_{2}^{2}}, \quad m_{1}^{*}=\frac{d_{2} k_{1} b_{1}}{d_{1} k_{2} b_{2}+d_{2} k_{1} b_{1}},
\end{aligned}
$$

$$
n_{1}^{*}=\frac{\left(r_{2} k_{2} b_{2}-\mu_{2} d_{2}\right) k_{1} b_{1}^{2}}{k_{2} b_{2}^{2} r_{1} k_{1} b_{1}-k_{2} b_{2}^{2} \mu_{1} d_{1}+k_{1} b_{1}^{2} r_{2} k_{2} b_{2}-k_{1} b_{1}^{2} \mu_{2} d_{2}} .
$$

through weak adaptations.

For illustration purpose, we consider the following example.

Example 4 We fix $r_{1}=3, r_{2}=2, \mu_{1}=1, \mu_{2}=1, b_{1}=1, b_{2}=0.5, k_{1}=$ $1, k_{2}=2.5, d_{1}=1, d_{2}=2, u=0.1, v=0.2, g=0.1, h=0.1$. Then $E^{*}=$ 
$(1,2,1.6,0.8,0.6154,0.2857)$. Thus, the capacity for the community of prey and predators in the first patch is $(1,2)$, and for the community in the second patch is $(1.6,0.8)$. Computer simulation shows that population densities in each patch approach the ideal state (see Figure 1). In contrast, if we remove the adaptations, and fix $m_{1}$ and $n_{1}$ as constants, for example, $m_{1}=n_{1}=0.5$, then densities of prey and predators in the first patch approach $(1.0521,1.9698)$, and densities of prey and predators in the second patch approach $(1.513,0.9436)$. Note that the fitness of prey is -0.0219 in the first patch and 0.0152 in the second patch, whereas the fitness of a predator is 0.052 in the first patch and -0.1087 in the second patch. Hence, the first patch is better for predators, but worse for prey, and the second patch is better for prey, but worse for predators. By contrast, the weak co-adaptations lead to the spatial distribution that there is no difference in patch fitness for both prey and predators by choosing $m_{1}^{*}=0.6154>0.5$ and $n_{1}^{*}=0.2857<0.5$. This means that an ideal free distribution for the prey population and the predator population has been achieved. In fact, the ideal free distribution for s single species, introduced by Fretwell and Lucas (1970), requires that all occupied patches have equal fitness. Further, the ideal free distribution for two species was defined by Cressman et al. (2004) as the distribution yielding equal fitness across occupied habitats for each species.

\section{Influences on persistence}

In this section, we study the influences of adaptations on persistence of populations in (3.4). We begin from the effect of adaptations on survival of predators. Let $m_{1}=m_{10}$ be the baseline transfer probability of prey from the first patch to the second patch when there is no fitness difference between the two patches for prey and $n_{1}=n_{10}$ be the baseline transfer probability of a predator from the first patch 
and is persistent if

$$
A=\left[\begin{array}{cc}
k_{1} b_{1} \bar{x}_{1}-d_{1}-v n_{10} & v\left(1-n_{10}\right) \\
v n_{10} & k_{2} b_{2} \bar{x}_{2}-d_{2}-v\left(1-n_{10}\right)
\end{array}\right],
$$

where $\left(\bar{x}_{1}, \bar{x}_{2}\right)$ is the unique positive equilibrium of the following system:

$$
\left\{\begin{array}{l}
\frac{d x_{1}}{d t}=x_{1}\left(r_{1}-\mu_{1} x_{1}-u m_{10}\right)+u\left(1-m_{10}\right) x_{2}, \\
\frac{d x_{2}}{d t}=x_{2}\left(r_{2}-\mu_{2} x_{2}-u\left(1-m_{10}\right)\right)+u m_{10} x_{1} .
\end{array}\right.
$$

We assume $0<m_{10}<1$ and $0<n_{10}<1$. By similar discussions to those in Wang and Zhao (2004, 2005a,b), we see that predators in (4.1) become extinct if

$$
\lambda_{\text {dom }}:=\operatorname{tr}(A)+\sqrt{(\operatorname{tr}(A))^{2}-4 \operatorname{det}(A)}<0,
$$

$$
\lambda_{\text {dom }}=\operatorname{tr}(A)+\sqrt{(\operatorname{tr}(A))^{2}-4 \operatorname{det}(A)}>0 .
$$


A key point that we should emphasize is that predators in (4.1) are either 252 persistent in both patches, or become extinct in both patches. This means that it 253 is impossible that predators survive in one patch and die out in the other patch, 254 which seems a contradiction to many ecological observations. Now, we show that

$$
\begin{aligned}
& E_{00 y}^{0}=\left(\frac{r_{1}}{\mu_{1}}, 0, \frac{r_{2}}{\mu_{2}}, 0, \frac{r_{2} \mu_{1}}{r_{1} \mu_{2}+r_{2} \mu_{1}}, 0\right), \\
& E_{00 y}^{1}=\left(\frac{r_{1}}{\mu_{1}}, 0, \frac{r_{2}}{\mu_{2}}, 0, \frac{r_{2} \mu_{1}}{r_{1} \mu_{2}+r_{2} \mu_{1}}, 1\right) .
\end{aligned}
$$

By direct calculations, we obtain

Theorem $5 E_{00 y}^{0}$ is stable if

$$
\begin{aligned}
& k_{1} b_{1} r_{1}<\mu_{1} d_{1}, \\
& k_{2} b_{2} r_{2}<\mu_{2}\left(d_{2}+v\right), \\
& k_{1} b_{1} \frac{r_{1}}{\mu_{1}}-d_{1}>k_{2} b_{2} \frac{r_{2}}{\mu_{2}}-d_{2},
\end{aligned}
$$

and is unstable if one of the inequalities in (4.4) is reversed.

Theorem $6 E_{00 y}^{1}$ is stable if

$$
\begin{aligned}
& k_{1} b_{1} r_{1}<\mu_{1}\left(d_{1}+v\right), \\
& k_{2} b_{2} r_{2}<\mu_{2} d_{2}, \\
& k_{1} b_{1} \frac{r_{1}}{\mu_{1}}-d_{1}<k_{2} b_{2} \frac{r_{2}}{\mu_{2}}-d_{2},
\end{aligned}
$$

and is unstable if one of the inequalities in (4.5) is reversed.

The stability of $E_{00 y}^{0}$ and $E_{00 y}^{1}$ presents opportunity that predators live well in a better habitat, but desert the worse one. Indeed, we first choose parameters such 
that $k_{1} b_{1} r_{1} / \mu_{1}-d_{1}<0, k_{2} b_{2} r_{2} / \mu_{2}-d_{2}-v<0$ and $k_{2} b_{2} r_{2} / \mu_{2}-d_{2}-\left(k_{1} b_{1} r_{1} / \mu_{1}-d_{1}\right)<$ 0 . Then we vary $k_{1}$ such that $k_{1} b_{1} r_{1} / \mu_{1}-d_{1}>0$, which leads to a stability transition of $E_{00 y}^{0}$ from a stable state to un unstable state. As a consequence, $E_{00 y}^{0}$ repels predators in the first patch away from extinction, at least locally, and another equilibrium

$$
E_{0 y}^{0}=\left(x_{1}^{*}, y_{1}^{*}, \frac{r_{2}}{\mu_{2}}, 0, \bar{m}_{1}, 0\right)
$$

emerges, where $x_{1}^{*}$ and $y_{1}^{*}$ are defined in (3.6) and

$$
\bar{m}_{1}=\frac{r_{2} / \mu_{2}}{x_{1}^{*}+r_{2} / \mu_{2}} \text {. }
$$

Numerical calculations show that $E_{0 y}^{0}$ is globally stable for a certain range of parameters, which confirms that predators can survive in the first patch and become extinct in the second patch in this case (see Figure 2).

Next, we show that the co-adaptation of prey and predators can enhance the survival probability of predators. To see this, we first select parameters such that

$$
\begin{aligned}
& k_{1} b_{1} \frac{r_{1}}{\mu_{1}}-d_{1}>0, \\
& k_{2} b_{2} \frac{r_{2}}{\mu_{2}}-d_{2}<0 .
\end{aligned}
$$

Note that (4.6) implies that $n_{1}$ decreases and converges to 0 as $t$ increases in a small neighborhood of $E_{0 y}^{0}$. By using the Routh-Hurwitz criteria, we can obtain the following theorem for the stability of $E_{0 y}^{0}$, the proof of which is postponed to Appendix.

Theorem $7 E_{0 y}^{0}$ is asymptotically stable if (4.6) holds and $g$ is small.

Theorem 7 supports the persistence of predators in the first patch and the extinction of predators in the second patch. On the other hand, if there is no adaptation of prey and predators, the persistence and extinction of predators are 
determined by the sign of $\lambda_{d o m}$. Note that in a small neighborhood of $v=0$, we have

$$
\lambda_{\text {dom }}=\frac{1}{2}\left[k_{1} b_{1} \bar{x}_{1}-d_{1}+k_{2} b_{2} \bar{x}_{2}-d_{2}-n_{10} v+o(v)\right],
$$

which suggests that $\lambda_{\text {dom }}$ may be a decreasing function of $v$, and may be negative for large $v$. To confirm this, we fix $r_{1}=1, r_{2}=1, \mu_{1}=2, \mu_{2}=1, d_{1}=1, d_{2}=$ $2, b_{1}=1, b_{2}=1, k_{1}=2.1, k_{2}=1, u=1, m_{10}=0.5, n_{10}=0.6$. Then $\bar{x}_{1}=0.6036$ and $\bar{x}_{2}=0.8536$, and

$$
\lambda_{\text {dom }}=-0.4395-0.5 v+0.5 \sqrt{1.9991-0.5656 v+v^{2}} .
$$

The graph of $\lambda_{d o m}$ with respective to $v$ is given in Figure 3, which shows that the dominant eigenvalue $\lambda_{d o m}$ of $A$ is a decreasing function of $v$ and $\lambda_{d o m}<0$ for larger $v$. Thus, without any adaptation of prey and predators, the increase of the maximal potential migration rate $v$ of predators from the first patch to the second patch tends to reduce the survival probability of predators, and leads to the extinction of predators after a threshold value. However, by reviewing the proof of Theorem 7, we see that the stability of $E_{0 y}^{0}$ is not influenced by the magnitude of $v$. Thus, we can fix $v$ such that $\lambda_{\text {dom }}<0$ and then choose small $g$, the adaptation coefficient of prey, such that $E_{0 y}^{0}$ is stable, which is possible as the second inequality in (4.6) holds. It follows that predators survive in the first patch. Therefore, the adaptation of prey and predators increases the survival probability of predators from the extinction in both patches to the persistence in one patch. Biological mechanism behind this phenomenon is that predators exploit better patches to escape extinction through adaptations. Nevertheless, Figure 3 also shows the possibility that predators can survive in both patches for small $v$ under the assumptions that there is no adaptation, but can survive only in the first patch with adaptations. The reason for the latter is that the first patch is better than the second patch for 
predators and adaptations make predators aggregate in the first patch.

We now present an example to show that there exists a pattern that prey and predators cooperate well through adaptations such that predators are permanent in every patch in the case that predators become extinct in each patch in the absence of adaptations. We take $r_{1}=1.2, r_{2}=1, \mu_{1}=2, \mu_{2}=1, d_{1}=1, d_{2}=2, b_{1}=1, b_{2}=$ $1, k_{1}=2, k_{2}=2.1, u=1, n_{10}=0.4, v=4$. By numerical calculations we obtain the graph of $\lambda_{d o m}$ versus $m_{10}$ in Figure 4 , which indicates $\lambda_{d o m}<0$ when $m_{10}$ is close to 0 or close to 1 . Thus, if there is no adaptation of prey and predators, predators become extinct in each patch when $m_{10}$ is close to 0 or close to 1 . If the adaptation of predators emerges and the adaptation of prey is absent, numerical calculations show that predators survive in the first patch for small $m_{10}$, and survive in the second patch when $m_{10}$ is close to 1 . But predators become extinct in the second patch for the former case, and become extinct in the first patch for the latter case (see Figure 5). Hence, the benefit of adaptation of only predators is to facilitate the survival in one patch. However, if both predators and prey adopt the adaptation strategies, things are different because the parameter values ensure that the full model with the adaptation of prey and predators admits a positive equilibrium. Further numerical simulations indicate that the positive equilibrium is globally stable (see Figure 6). Hence, prey and predators coordinate well so that predators survive in every patch. We now explain why there exist such patterns through the adaptations. First, in the absence of prey adaptation, predators test the better patch to migrate to that patch to survive. For example, if we fix $m_{10}=0.1$ and keep other parameters as above, the first patch is better for predators through less prey emigration from the first patch and more prey migration to the first patch. In fact, with the adaptation of predators, the fitness of a predator tends to 0 in the first patch and -1.41389 in the second patch. Although predators can survive in the first patch in this case, the fitness of prey is -0.4024 in the first patch and 0.7209 in 
the second patch. This means that the second patch is better for prey, the reason of which is that the prey in the first patch faces more predation pressure. Thus, if the adaptation of prey is allowed, more prey will migrate to the second patch. As a result, the fitness of a predator increases in the second patch and decreases in the first patch. This encourages a fraction of predators to migrate to the second patch so that the coexistence patter of prey and predators occurs.

\section{Behaviors from strong response}

In this section, by considering (3.4) we show that strong adaptations of prey and predators may induce complicated dynamical behaviors.

First, equilibrium $E_{0 y}^{0}$ exhibits stability transitions as the adaptation coefficient $g$ increases, which suggests the possibility of a Hopf bifurcation. This is confirmed by numerical simulations. For the parameters with larger $r_{1}$ and $g$ in Figure 7 , prey's densities in the two patches approach periodic cycles, and predators always stay in the first patch, but exhibit periodic fluctuations after a transient time. Biological reason for this type of cycles could be given as follows. Rich prey in the first patch attracts a large number of predators, which in turn give the higher predation pressure on prey. Then a strong response leads to a quick migration of prey to the second patch, which decreases the prey density in the first patch. In the process, predators always stay in the first patch as the large $r_{1}$ leads to rich prey in the first patch, which implies that the fitness of a predator in the first patch is higher. Thus, the decrease of prey reduces predators in the first patch. The cycles emerge from repetitions of the processes. In contrast, in the absence of the adaptations, simulations with the parameters given in Figure 7 indicate that populations in each patch approach to globally stable states with $x_{1}=1.7767, x_{2}=0.4893, y_{1}=5.8610, y_{2}=2.8262$ (see Figure 8). Hence, the 
adjustment of prey to equality in patch fitness destroys the stable coexistence of ecological community of prey and predators in each patch, and leads to periodic cycles.

We now consider influences of strong adaptations on the stability of positive equilibrium $E^{*}$. For larger $g$ and $h, E^{*}$ becomes unstable in several cases. First, $E^{*}$ becomes unstable for a larger intrinsic growth rate of prey (see the left panel in Figure 9). Secondly, there exists a region of $u$ and $v$, which is similar to a bottle, such that $E^{*}$ is unstable inside the region and is stable outside the region (see the right panel in Figure 9). Variations of $k_{1}$ and $k_{2}$, or $b_{1}$ and $b_{2}$, exhibit similar influences on the stability of $E^{*}$. Clearly, the figures show that there are multiple stability switches if we fix one parameter and vary the other. For example, for the case described by the left panel of Figure 9 , if we fix $r_{1}=3$ and vary $r_{2}$, then there are Hopf bifurcations when $r_{2}=1.7357, r_{2}=6.0363$ and $r_{2}=11.7166$. $E^{*}$ is unstable when $r_{2}$ lies in $(1.7357,6.0363)$ and $r_{2}>11.7166$. There are a family of stable periodic solutions when $r_{2}$ varies in $(1.7357,6.0363)$ and a family of stable periodic solutions when $r_{2}$ varies in $r_{2}>11.7166$ (see Figure 10).

\section{Discussions}

In this paper, we have proposed a prey-predator model that incorporates densitydependent migrations through the adaptations of prey and predators between the two patches. We have assumed that a movable individual decides its migration probability in terms of the law that the rate of migration probability is proportional to the difference of the fitness of the destination patch and the average fitness. This modeling method, based upon the assumption that an individual has the ability to know the fitness in each patch, gives an alternative migration rule and allows more mathematical analysis to find more interesting phenomena. 
In Theorem 1 and Theorem 2, we have shown that prey evolves, through weak adaptation, to the ideal spatial distribution where the carrying capacity of prey population in each patch is achieved. By means of analytic method in Theorem 3 and simulations, we have verified that the full system of prey-predator interactions with weak adaptations and small migration amplitudes implies that the ideal spatial distribution is established in the sense that the carrying capacity of prey-predator community in each patch is approached.

For classical patchy models of prey and predators without adaptations, either predator population survives in every patch, or becomes extinct in every patch unless there is no barrier to predators between two patches. With the introduction of the adaptations, we have shown that the predator population can survive only in the better patch, and desert the worse one. This means that there is no need to use barriers to limit predators in a favorable patch if predators are able to adapt. We have also analyzed that the adaptation of prey and predators increases the survival probability of predators from the extinction in both patches to the persistence in one patch. Furthermore, we have presented the example that prey and predators cooperate well through adaptations to ensure the permanence of predators in every patch in the case that predators become extinct in each patch in the absence of adaptations.

For the strong responses of prey and predators, we have shown that the adjustment of prey to establish equality in patch fitness destroys the stable coexistence of ecological community of prey and predators in each patch, and leads to periodic cycles. As a result, the objective of equal fitness in every patch cannot be realized. Moreover, we have presented the stability regions for large adaptations that show the possibility of multiple stability transitions if we vary one parameter. 


\section{${ }_{412}$ Acknowledgments}

${ }_{413}$ The authors would like to thank anonymous referees for very helpful suggestions and comments which led to improvements of our original manuscript.

\section{Appendix}

\section{Proof of Theorem 1}

To find a positive equilibrium, we set the right-hand side of the third equation of (3.1) to 0 to obtain

$$
g_{2}\left(x_{2}\right)=g_{1}\left(x_{1}\right) .
$$

It follows from the first equation and the second equation of (3.1) that

$$
u\left(1-m_{1}\right)-u m_{1} \frac{x_{1}}{x_{2}}=u m_{1}-u\left(1-m_{1}\right) \frac{x_{2}}{x_{1}},
$$

which leads to

$$
m_{1}=\frac{x_{2}}{x_{1}+x_{2}} .
$$

migration rate of prey to patch $1=u\left(1-m_{1}\right) x_{2}=u \frac{x_{1} x_{2}}{x_{1}+x_{2}}$,

emigration rate of prey from patch $1=u m_{1} x_{1}=u \frac{x_{1} x_{2}}{x_{1}+x_{2}}$.

It follows that the inflow rate of prey to patch 1 equals the outflow rate of prey from patch 1 . Then it is easy to see that $E_{\text {prey }}$ is the unique positive equilibrium of (3.1). The proof is complete.

\section{Proof of Theorem 2}

We begin by showing that $E_{\text {prey }}$ is asymptotically stable. The Jacobian matrix 
of (3.1) at $E_{\text {prey }}$ is

$$
\left[\begin{array}{ccc}
-u m_{1}^{*}+C_{1} g_{1}^{\prime}\left(C_{1}\right) & u\left(1-m_{1}^{*}\right) & -u\left(C_{1}+C_{2}\right) \\
u m_{1}^{*} & -u\left(1-m_{1}^{*}\right)+C_{2} g_{2}^{\prime}\left(C_{2}\right) & u\left(C_{1}+C_{2}\right) \\
-g m_{1}^{*}\left(1-m_{1}^{*}\right) g_{1}^{\prime}\left(C_{1}\right) & g m_{1}^{*}\left(1-m_{1}^{*}\right) g_{2}^{\prime}\left(C_{2}\right) & 0
\end{array}\right]
$$

435

Its characteristic equation is

$$
\lambda^{3}+a_{1} \lambda^{2}+a_{2} \lambda+a_{3}=0,
$$

where

$$
\begin{aligned}
a_{1}= & -C_{1} g_{1}^{\prime}\left(C_{1}\right)-C_{2} g_{2}^{\prime}\left(C_{2}\right)+u>0, \\
a_{2}= & -g u m_{1}^{*}\left(C_{1}+C_{2}\right)\left(1-m_{1}^{*}\right)\left(g_{1}^{\prime}\left(C_{1}\right)+g_{2}^{\prime}\left(C_{2}\right)\right) \\
& +C_{1} C_{2} g_{1}^{\prime}\left(C_{1}\right) g_{2}^{\prime}\left(C_{2}\right)-u C_{1} g_{1}^{\prime}\left(C_{1}\right)\left(1-m_{1}^{*}\right)-u m_{1}^{*} C_{2} g_{2}^{\prime}\left(C_{2}\right)>0, \\
a_{3}= & u m_{1}^{*} g g_{1}^{\prime}\left(C_{1}\right) g_{2}^{\prime}\left(C_{2}\right)\left(C_{1}+C_{2}\right)^{2}\left(1-m_{1}^{*}\right)>0 .
\end{aligned}
$$

Set

$$
Q_{1}=u m_{1}^{*}\left(C_{1}+C_{2}\right)\left(1-m_{1}^{*}\right)\left(\left(g_{1}^{\prime}\left(C_{1}\right)\right)^{2} C_{1}+C_{2}\left(g_{2}^{\prime}\left(C_{2}\right)\right)^{2}-u\left(g_{1}^{\prime}\left(C_{1}\right)+g_{2}^{\prime}\left(C_{2}\right)\right)\right),
$$

$$
\begin{aligned}
Q_{2}= & \left(u-C_{1} g_{1}^{\prime}\left(C_{1}\right)-C_{2} g_{2}^{\prime}\left(C_{2}\right)\right)\left(C_{1} g_{1}^{\prime}\left(C_{1}\right) C_{2} g_{2}^{\prime}\left(C_{2}\right)\right. \\
& \left.-u m_{1}^{*} C_{2} g_{2}^{\prime}\left(C_{2}\right)-u C_{1} g_{1}^{\prime}\left(C_{1}\right)\left(1-m_{1}^{*}\right)\right) .
\end{aligned}
$$

Then direct calculations lead to

$$
a_{1} a_{2}-a_{3}=g Q_{1}+Q_{2}>0 .
$$

It follows from the Hurwitz criteria that $E_{\text {prey }}$ is asymptotically stable.

Now, we use techniques of singular perturbation theory to show that each pos- 
${ }_{448} J\left(x_{1}, x_{2}, m_{1}\right)=\left(\begin{array}{cc}g_{1}\left(x_{1}\right)-u m_{1}+x_{1} g_{1}^{\prime}\left(x_{1}\right) & u\left(1-m_{1}\right) \\ u m_{1} & g_{2}\left(x_{2}\right)-u\left(1-m_{1}\right)+x_{2} g_{2}^{\prime}\left(x_{2}\right)\end{array}\right)$.

where prime denotes the derivative with respect to $\tau$. Since $g$ is a small parameter, (A-2) is a slow system and (3.1) is a fast system. The slow manifold is determined by

$$
\begin{aligned}
& F_{1}\left(x_{1}, x_{2}, m_{1}\right):=x_{1}\left(g_{1}\left(x_{1}\right)-u m_{1}\right)+u\left(1-m_{1}\right) x_{2}=0, \\
& F_{2}\left(x_{1}, x_{2}, m_{1}\right):=x_{2}\left(g_{2}\left(x_{2}\right)-u\left(1-m_{1}\right)\right)+u m_{1} x_{1}=0 .
\end{aligned}
$$

Note that

$$
g_{1}\left(x_{1}\right)-u m_{1}=-u\left(1-m_{1}\right) \frac{x_{2}}{x_{1}},
$$

$$
g_{2}\left(x_{2}\right)-u\left(1-m_{1}\right)=-u m_{1} \frac{x_{1}}{x_{2}} .
$$

451 It follows from $g_{i}^{\prime}\left(x_{i}\right)<0$ that

${ }_{452} \operatorname{det} J\left(x_{1}, x_{2}, m_{1}\right)=-u\left(1-m_{1}\right) g_{2}^{\prime}\left(x_{2}\right) \frac{x_{2}^{2}}{x_{1}}-u m_{1} g_{1}^{\prime}\left(x_{1}\right) \frac{x_{1}^{2}}{x_{2}}+x_{1} x_{2} g_{1}^{\prime}\left(x_{1}\right) g_{2}^{\prime}\left(x_{2}\right)>0$.

${ }_{453}$ Since $F_{1}=0$ and $F_{2}=0$ at $E_{\text {prey }}$, it follows from the implicit function theorem 
Set

$$
J_{x_{1}}=\left(\begin{array}{cc}
-u\left(C_{1}+C_{2}\right) & u\left(1-m_{1}^{*}\right) \\
u\left(C_{1}+C_{2}\right) & -u\left(1-m_{1}^{*}\right)+C_{2} g_{2}^{\prime}\left(C_{2}\right)
\end{array}\right)
$$

and

$$
J_{x_{2}}=\left(\begin{array}{cc}
-u m_{1}^{*}+C_{1} g_{1}^{\prime}\left(C_{1}\right) & -u\left(C_{1}+C_{2}\right) \\
u m_{1}^{*} & u\left(C_{1}+C_{2}\right)
\end{array}\right)
$$

with $m_{1}^{*}=C_{2} /\left(C_{1}+C_{2}\right)$. Then $\operatorname{det} J_{x_{1}}>0$ and $\operatorname{det} J_{x_{2}}<0$. Hence, on $S$ we have

$$
x_{1}^{\prime}\left(m_{1}^{*}\right)=-\operatorname{det} J_{x_{1}} / \operatorname{det} J\left(C_{1}, C_{2}, m_{1}^{*}\right)<0,
$$

$$
x_{2}^{\prime}\left(m_{1}^{*}\right)=-\operatorname{det} J_{x_{2}} / \operatorname{det} J\left(C_{1}, C_{2}, m_{1}^{*}\right)>0 .
$$

If we denote the right-hand side of the third equation of (A-2) by $F_{3}\left(x_{1}, x_{2}, m_{1}\right)$, it follows that $F_{3}\left(x_{1}\left(m_{1}\right), x_{2}\left(m_{1}\right), m_{1}\right)$ satisfies

$$
F_{3} \begin{cases}<0 & \text { if } m_{1}^{*}<m_{1}<m_{1}^{*}+\epsilon \\ >0 & \text { if } m_{1}^{*}>m_{1}>m_{1}^{*}-\epsilon\end{cases}
$$

for some positive $\epsilon$. From the argument in the proof of Theorem 1 we see that $F_{3}\left(x_{1}\left(m_{1}\right), x_{2}\left(m_{1}\right), m_{1}\right)$ can not be zero for $m_{1}>m_{1}^{*}$ or $m_{1}<m_{1}^{*}$. Therefore, equilibrium $m_{1}=m_{1}^{*}$ is globally asymptotically stable on $S$.

We consider a subsystem of (3.1):

$$
\left\{\begin{array}{l}
x_{1}^{\prime}=x_{1}\left(g_{1}\left(x_{1}\right)-u m_{1}\right)+u\left(1-m_{1}\right) x_{2}, \\
x_{2}^{\prime}=x_{2}\left(g_{2}\left(x_{2}\right)-u\left(1-m_{1}\right)\right)+u m_{1} x_{1} .
\end{array}\right.
$$

By the monotonic flow techniques (Lu and Takeuchi, 1993; Smith, 1995; Zhao, $2003)$ we see that the positive equilibrium $\left(x_{1}\left(m_{1}\right), x_{2}\left(m_{1}\right)\right)$ is globally stable in the interior of $R_{+}^{2}$ for (3.1). Thus, given $\epsilon>0$, for any interval $M_{0}:=\left[m_{00}, m^{00}\right]$ 
471 with $0<m_{00}<m_{1}^{*}<m^{00}<1$, and a compact set $X_{0}$ in the interior of $R_{+}^{2}$, there 472 is a $T_{0}>0$ such that any positive solution $\left(x_{1}(t), x_{2}(t)\right)$ of $(\mathrm{A}-4)$ staring in $X_{0}$ with ${ }_{473} m_{00} \leq m_{1} \leq m^{00}$ satisfies

$$
\left|x_{i}(t)-x_{i}(m)\right|<\epsilon, \quad i=1,2, \quad t \geq T_{0} .
$$

474 Note that $E_{\text {prey }}$ is the unique positive equilibrium of (3.1) and is asymptotically 475 stable. It follows from the theory of geometric singular perturbation (Fenichel, 1979; Jones, 1994) that $E_{\text {prey }}$ is globally stable for small adaptation coefficient $g$.

477 The proof is complete.

$478 \quad$ Proof of Theorem 7:

${ }_{479}$ The characteristic equation of the Jacobian matrix of (3.4) at $E_{0 y}^{0}$ has 2 roots:

$$
\begin{aligned}
& \lambda_{1}=k_{2} b_{2} \frac{r_{2}}{\mu_{2}}-d_{2}-v<0, \\
& \lambda_{2}=h\left(k_{2} b_{2} \frac{r_{2}}{\mu_{2}}-d_{2}\right)<0 .
\end{aligned}
$$

The other roots satisfy the following equation

$$
A_{0} \lambda^{4}+A_{1} \lambda^{3}+A_{2} \lambda^{2}+A_{3} \lambda+A_{4}=0,
$$


where

$$
\begin{aligned}
A_{0}= & b_{1} k_{1}\left(d_{1} \mu_{2}+r_{2} k_{1} b_{1}\right), \\
A_{1}= & \left(d_{1} \mu_{2}+r_{2} k_{1} b_{1}\right)\left(r_{2} k_{1} b_{1}+b_{1} u k_{1}+\mu_{1} d_{1}\right), \\
A_{2}= & g b_{1} r_{2} k_{1} d_{1} u\left(\mu_{2}+\mu_{1}\right)+\mu_{1} d_{1}\left(-d_{1}^{2} \mu_{2}+u d_{1} \mu_{2}-d_{1} r_{2} k_{1} b_{1}+r_{2} d_{1} \mu_{2}+r_{2}^{2} k_{1} b_{1}\right) \\
& +b_{1} k_{1}\left(k_{1} b_{1} r_{2} d_{1} r_{1}+b_{1} r_{2}^{2} k_{1} u+d_{1}^{2} \mu_{2} r_{1}\right), \\
A_{3}= & d_{1} g u r_{2}\left(b_{1} k_{1} \mu_{1} r_{2}-k_{1} b_{1} \mu_{1} d_{1}+\mu_{1} \mu_{2} d_{1}+k_{1}^{2} b_{1}^{2} r_{1}\right) \\
& +d_{1}\left(r_{2} d_{1} \mu_{2}+r_{2}^{2} k_{1} b_{1}+u d_{1} \mu_{2}\right)\left(r_{1} k_{1} b_{1}-\mu_{1} d_{1}\right), \\
A_{4}= & u r_{2} d_{1} g\left(d_{1} \mu_{2}+r_{2} k_{1} b_{1}\right)\left(r_{1} k_{1} b_{1}-\mu_{1} d_{1}\right) .
\end{aligned}
$$

Evidently, $A_{0}>0, A_{1}>0$ and $A_{4}>0$. By the Routh-Hurwitz criteria, we need to consider the signs:

$$
D_{2}=A_{1} A_{2}-A_{0} A_{3}, \quad D_{3}=\left|\begin{array}{ccc}
A_{1} & A_{3} & 0 \\
A_{0} & A_{2} & A_{4} \\
0 & A_{1} & A_{3}
\end{array}\right| .
$$

By direct calculations, we see that the sign of $D_{2}$ is determined by

$$
\begin{aligned}
D_{20}= & g u r_{2} k_{1} d_{1} b_{1}\left(-r_{1} k_{1}^{2} b_{1}^{2}+k_{1} b_{1} u \mu_{2}+k_{1} b_{1} u \mu_{1}+r_{2} k_{1} b_{1} \mu_{2}+\mu_{1} k_{1} b_{1} d_{1}+d_{1} \mu_{1}^{2}\right) \\
& +\varphi_{0}\left(\mu_{1}, \mu_{2}, r_{1}, r_{2}, k_{1}, b_{1}, b_{2}, u, d_{1}\right)
\end{aligned}
$$

where

$$
\begin{aligned}
\varphi_{0}= & r_{2}^{3} b_{1}^{2} k_{1}^{2}\left(b_{1} k_{1} u+\mu_{1} d_{1}\right)+r_{2}^{2} b_{1} k_{1}\left(u^{2} b_{1}^{2} k_{1}^{2}+2 b_{1} d_{1} u \mu_{1} k_{1}+\mu_{1}^{2} d_{1}^{2}+\mu_{1} d_{1}^{2} \mu_{2}\right) \\
& +r_{2} d_{1}\left(-b_{1} d_{1}^{2} \mu_{1}^{2} k_{1}+\mu_{1}^{2} d_{1}^{2} \mu_{2}+d_{1} b_{1}^{2} \mu_{1} k_{1}^{2} r_{1}-b_{1}^{2} d_{1} u k_{1}^{2} \mu_{1}+2 u k_{1} b_{1} d_{1} \mu_{2} \mu_{1}+b_{1}^{3} u k_{1}^{3} r_{1}\right) \\
& +\mu_{1} d_{1}^{2} \mu_{2}\left(-d_{1}^{2} \mu_{1}+u d_{1} \mu_{1}+b_{1} d_{1} k_{1} r_{1}+b_{1} k_{1} u^{2}\right) .
\end{aligned}
$$

481 Since $k_{1} b_{1} \frac{r_{1}}{\mu_{1}}>d_{1}$, it is easy to see $\varphi_{0}>0$. It follows that $D_{20}$ is positive if $g$ is 
small, and therefore $D_{2}$ is positive if $g$ is small.

The sign of $D_{3}$ is determined by

$$
\begin{aligned}
D_{30}= & g^{2}\left(-r_{1} k_{1}^{2} b_{1}^{2}+k_{1} b_{1} u \mu_{2}+k_{1} b_{1} u \mu_{1}+r_{2} k_{1} b_{1} \mu_{2}+\mu_{1} k_{1} b_{1} d_{1}+d_{1} \mu_{1}^{2}\right) \varphi_{2} \\
& +g \varphi_{1}+\varphi_{0}\left(\mu_{1}, \mu_{2}, r_{1}, r_{2}, k_{1}, b_{1}, b_{2}, u, d_{1}\right)
\end{aligned}
$$

where $\varphi_{1}$ and $\varphi_{2}$ are polynomials of parameters of the model without $g, k_{2}, c_{2}, v$.

Hence, $D_{3}$ is positive when $g$ is small. Consequently, the Routh-Hurwitz criteria imply that all roots of (A-7) admit negative real parts.

\section{References}

A. E. Abdllaoui, P. Auger, B. W. Kooi, R. B. de la Parra, and R. Mchich. Effects of density-dependent migrations on stability of a two-patch predator-prey model. Math. Biosci., 210:335-354, 2007.

P. A. Abrams. Habitat choice in predator-prey systems: spatial instability due to interacting adaptive movements. American Naturalist, 169:581-594, 2007.

P. A. Abrams. The impact of habitat selection on the heterogeneity of resources in varying environments. Ecology, 81:2902-2913, 2000.

P. A. Abrams. Can adaptive evolution or behavior lead to diversification of traits determining a trade-off between foraging gain and predation risk? Evolutionary Ecology Research, 5:653-670, 2003.

P. A. Abrams and H. Matsuda. Consequences of behavioral dynamics for the population dynamics of predator-prey systems with switching. Population Ecology, 46:13-25, 2004. 
P. A. Abrams, R. Cressman, and V. Krivan. The role of behavioral dynamics in determining the patch distributions of interacting species. American Naturalist, 169:505-518, 2007.

J. Arino and P. van den Driessche. Disease spread in metapopulations. In Nonlinear dynamics and evolution equations, pages 1-12. Fields Inst. Commun. 48, Amer. Math. Soc., Providence, RI, 2006.

C. Bernstein. Prey and predator emigration responses in the acarine system. Oecologia, 61:134-142, 1984.

C. Briggs and M. Hoopes. Stabilizing effects in spatial parasitoidchost and predatorprey models: a review. Theoretical Population Biology, 65:299-315, 2004.

G. Chiorino, P. Auger, J. L. Chassé, and S. Charles. Behavioral choices based on patch selection: a model using aggregation methods. Math. Biosci., 157:189-216, 1999.

R. Cressman and V. Krivan. Migration dynamics for the ideal free distribution. American Naturalist, 168:384-397, 2006.

R. Cressman, V. Krivan, and J. Garay. Ideal free distributions, evolutionary games, and population dynamics in multiple-species environments. American Naturalist, 164:473-489, 2004.

A. M. de Roos, K. Leonardsson, L. Persson, and G. G. Mittelbach. Ontogenetic niche shifts and flexible behavior in size-structured populations. Ecological Monographs, 72:271-292, 2002.

D. L. DeAngelis, M. Vos, W. M. Mooij, and P. A. Abrams. Feedback effects between the food chain and induced defense strategies. Fundamental ecology: from energetics to food webs. Springer, Netherlands, 2006. 
U. Diekmann, R. Law, and J. A. J. Meta. The geometry of ecological interactions: Simplifying spatial complexity. Oxford University Press, Oxford, 2000.

N. Fenichel. Geometric singular perturbation theory for ordinary differential equations. J. Diff. Eqns., 31:53-98, 1979.

D. Fonseca and D. Hart. Density-dependent dispersal of black fly neonates is mediated by flow. Oikos, 75:49-58, 1996.

D. R. French and J. Travis. Density-dependent dispersal in host-parasitoid assemblages. Oikos, 95:125-135, 2001.

S. D. Fretwell and H. L. Lucas. On territorial behavior and other factors influencing habitat distribution in birds. Acta Biotheoretica, 19:16-36, 1970.

I. Hanski. Metapopulation dynamics. Nature, 396:41-49, 1998.

A. Hastings. Can spatial variation alone lead to selection for dispersal? Theor. Pop. Biol., 24:244-251, 1983.

C. Hauzy, F. D. Hulot, A. Gins, and M. Loreau. Intra- and interspecific densitydependent dispersal in an aquatic preycpredator system. J. Anim. Ecol., 76: $552-558,2007$.

J. Hofbauer and K. Sigmund. Evolutionary Games and Population Dynamics. Cambridge University Press, 1998.

C. Jones. Geometric singular perturbation theory. In Dynamical Systems (Montecatini. Terme, 1994), Lect. Notes in Math. 1609. Springer, Berlin, 1994.

E. Kisdi and S. Liu. Evolution of handling time can destroy the coexistence of cycling predators. J. Evol. Biol., 19:49-58, 2006. 
Y. Kuang and Y. Takeuchi. Predator-prey dynamics in models of prey dispersal in two-patch environments. Math. Biosci., 120:77-98, 1994.

S. Levin. Dispersion and population interactions. American Naturalist, 108:207228,1974 .

Y. Lou. Some challenging mathematical problems in evolution of dispersal and population dynamics. In A. Friedman, editor, Tutorials in mathematical biosciences. IV, Evolution and ecology. Springer-Verlag, Berlin, 2008.

Z. Lu and Y. Takeuchi. Global asymptotic behavior in single-species discrete diffusion systems. J. Math. Biol., 32:67-77, 1993.

E. Matthysen. Density-dependent dispersal in birds and mammals. Ecography, 28: 403-416, 2005.

R. Mchich, P. Auger, and J. C. Poggiale. Effect of predator density dependent dispersal of prey on stability of a predator-prey system. Math. Biosci., 206: 343-356, 2007.

J. D. Murray. Mathematical biology. II. Spatial models and biomedical applications. Third edition. Springer-Verlag, New York, 2003.

T. Namba, A. Umemoto, and E. Minami. The effects of habitat fragmentation on persistence of source-sink metapopulations in systems with predators and prey or apparent competitors. Theoretical Population Biology, 56:123-137, 1999.

L. Persson and A. M. de Roos. Adaptive habitat use in size-structured populations: linking individual behavior to population processes. Ecology, 84:1129-1139, 2003.

N. Shigesada and K. Kawasaki. Biological invasions: theory and practice. Oxford University Press, Oxford, 1997. 
H. L. Smith. Monotone dynamical systems. An introduction to the theory of competitive and cooperative systems. American Mathematical Society, Providence, RI, 1995.

Y. Takeuchi. Diffusion effect on stability of lotka-volterra model. Bull. Math. Biol., 46:585-601, 1986.

Y. Takeuchi, Y. Iwasa, and K. Sato. Mathematics for life sciences and medicine. Springer-Verlag, Berlin, 2007.

W. Wang and X. Zhao. An epidemic model in a patchy environment. Mathematical Biosciences, 190:97-112, 2004.

W. Wang and X. Zhao. An age-structured epidemic model in a patchy environment. SIAM J. Appl. Math., 65:1597-1614, 2005a.

W. Wang and X. Zhao. an epidemic model with population dispersal and infection period. SIAM J. Appl. Math., 66:1454-1472, 2005b.

A. Watkinson and W. Sutherland. Sources, sinks and pseudo-sinks. J. Anim. Ecol., 64:126-130, 1995.

X.-Q. Zhao. Dynamical Systems in Population Biology. Springer-Verlag, New York, 2003. 


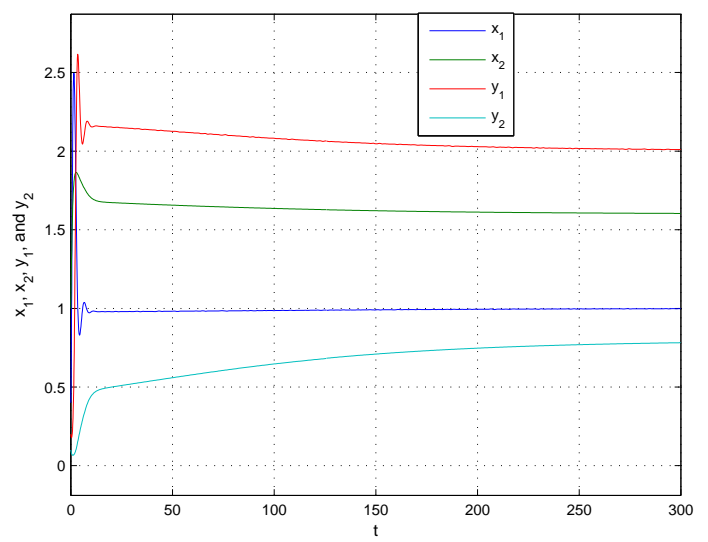

Figure 1: Prey and predators are adjusted to the carrying capacities of ecological community through weak adaptation. 

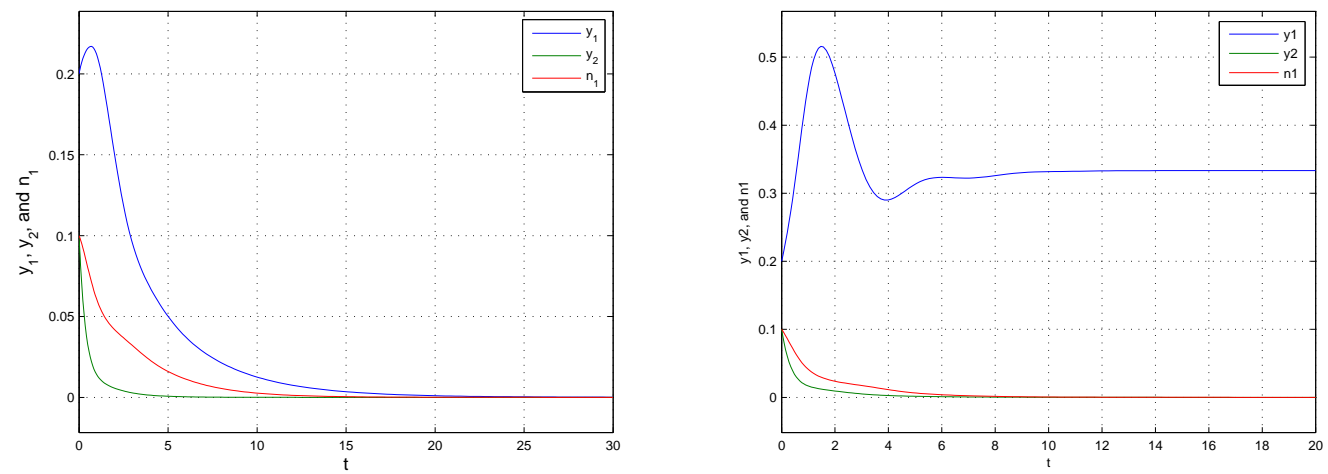

Figure 2: Left panel shows the extinction of predators in the two patches where the parameters are fixed as $r_{1}=1, r_{2}=1, \mu_{1}=2, \mu_{2}=1, u=1, v=2, k_{1}=$ $1.5, k_{2}=1, b_{1}=1, b_{2}=1, d_{1}=1, d_{2}=2, g=5, h=0.5$. The right panel shows that predators survive in the first patch, but become extinct in the second patch where the parameters are fixed as $r_{1}=1, r_{2}=1, \mu_{1}=2, \mu_{2}=1, u=1, v=2, k_{1}=$ $3, k_{2}=1, b_{1}=1, b_{2}=1, d_{1}=1, d_{2}=2, g=5, h=0.5$.

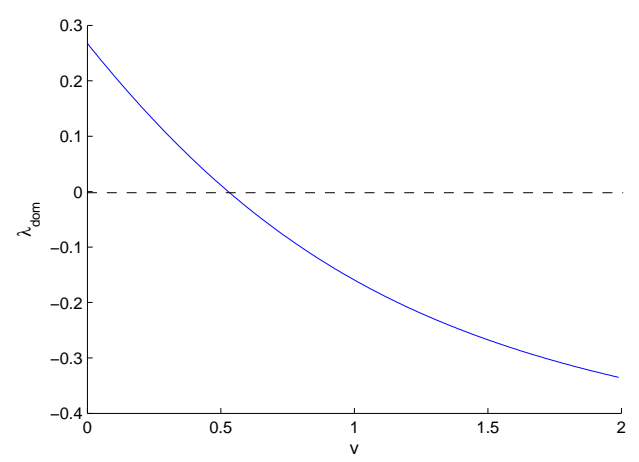

Figure 3: The graph of dominant eigenvalue $\lambda_{\text {dom }}$ versus $v$. 


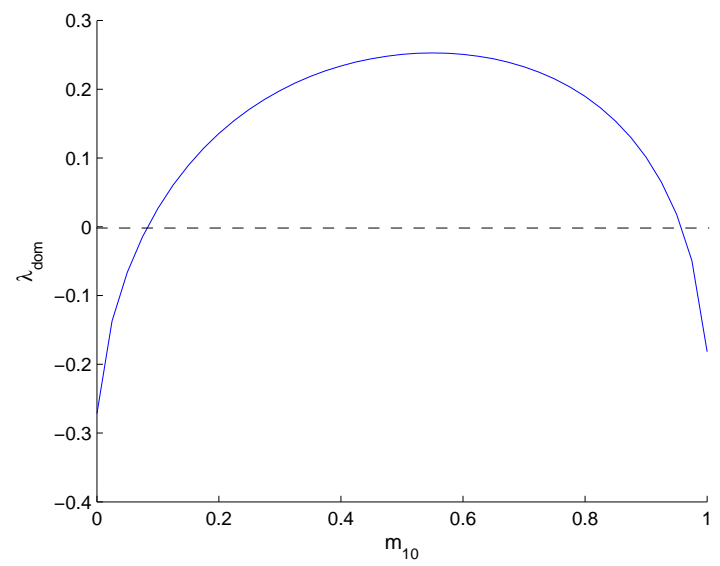

Figure 4: The graph of dominant eigenvalue $\lambda_{\text {dom }}$ versus $m_{10}$.
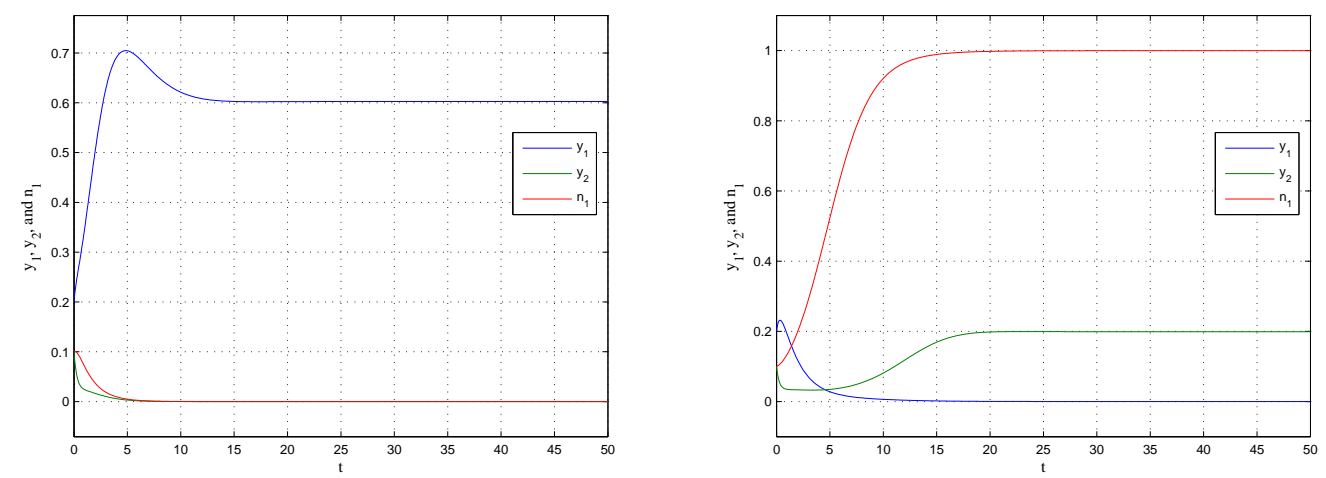

Figure 5: Left panel shows that the adaptation of only predators leads to the persistence of predators in the first patch for small $m_{10}$, where the parameters are fixed as $r_{1}=1.2, r_{2}=1, \mu_{1}=2, \mu_{2}=1, d_{1}=1, d_{2}=2, b_{1}=1, b_{2}=1, k_{1}=2, k_{2}=$ $2.1, u=1, v=4, h=0.5$ and $m_{10}=0.1$. The right panel shows that the adaptation of only predators leads to the persistence of predators in the second patch for $m_{10}$ close to 1 , where the parameters are fixed as $r_{1}=1.2, r_{2}=1, \mu_{1}=2, \mu_{2}=1, d_{1}=$ $1, d_{2}=2, b_{1}=1, b_{2}=1, k_{1}=2, k_{2}=2.1, u=1, v=4, h=0.5$ and $m_{10}=0.98$. 


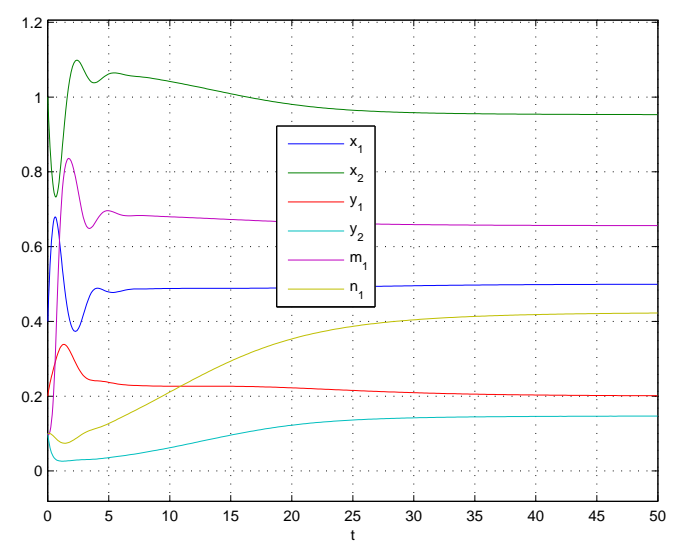

Figure 6: Predators survive in two patches where parameters are fixed as $r_{1}=$ $1.2, r_{2}=1.1, \mu_{1}=2, \mu_{2}=1, d_{1}=1, d_{2}=2, b_{1}=1, b_{2}=1, k_{1}=2, k_{2}=2.1, u=$ $1, v=4, g=5, h=0.5$.
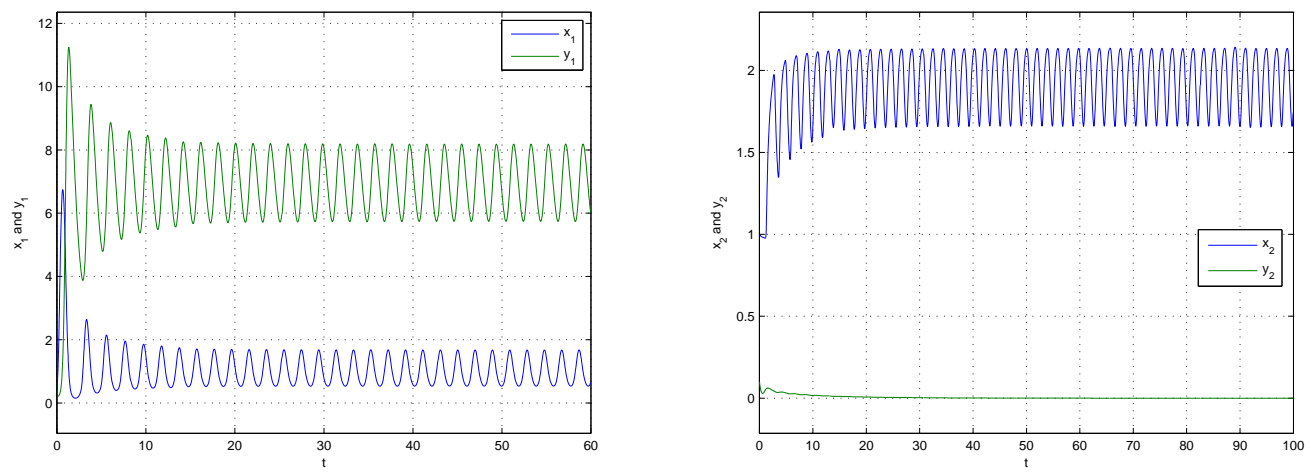

Figure 7: Left panel shows the periodic oscillations of prey and predators in the first patch and right panel indicates the periodic oscillation of prey and the extinction of predators in the second patch, where the parameters are fixed as $r_{1}=8, r_{2}=$ $2, d_{1}=1, d_{2}=2.1, \mu_{1}=1, \mu_{2}=1, u=1, v=3, k_{1}=1, k_{2}=1, b_{1}=1, b_{2}=1, g=$ $5, h=0.5$. 


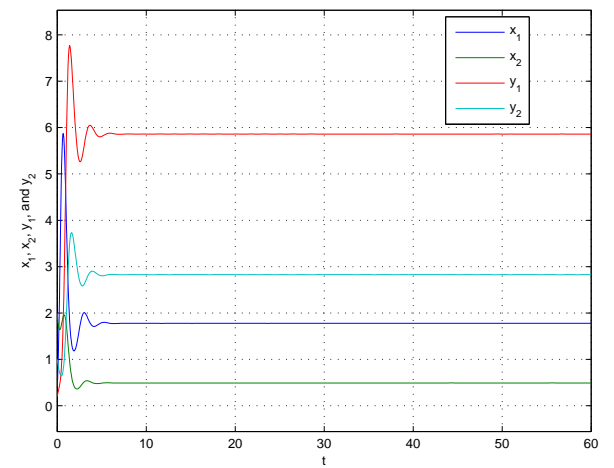

Figure 8: Prey and predators approach globally stable states when there is no adaptation for prey and predators, where parameters are fixed as $r_{1}=8, r_{2}=$ $2, d_{1}=1, d_{2}=2.1, \mu_{1}=1, \mu_{2}=1, u=1, v=3, k_{1}=1, k_{2}=1, b_{1}=1, b_{2}=1, m_{1}=$ $0.5, n_{1}=0.5$.
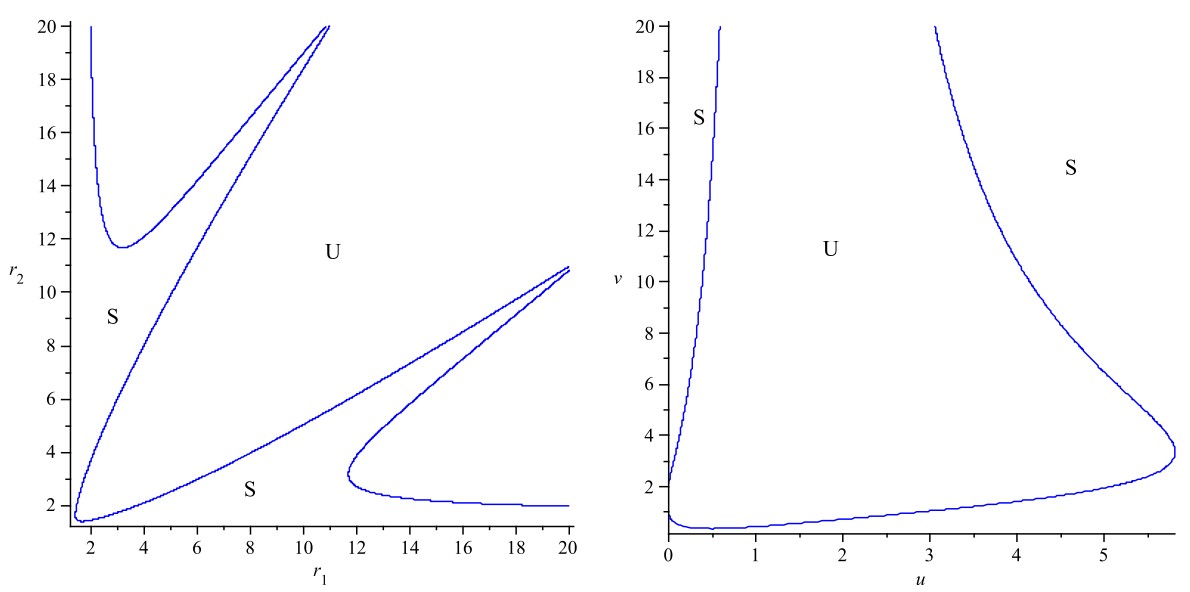

Figure 9: Left panel describes the curve below which $E^{*}$ is stable and above which $E^{*}$ is unstable, where $g=3, h=4, k_{1}=1, k_{2}=1, u=0.5, v=0.4, d_{1}=2, d_{2}=$ $2, b_{1}=2, b_{2}=2, \mu_{1}=1, \mu_{2}=1$, and right panel shows boundaries between stable region and unstable region where $k_{1}=1, k_{2}=1, r_{1}=2, r_{2}=1.5, g=3, h=4, d_{1}=$ $2, d_{2}=2, b_{1}=2, b_{2}=2, \mu_{1}=1, \mu_{2}=1$. $U$ represents an unstable region and $S$ represents a stable region. 


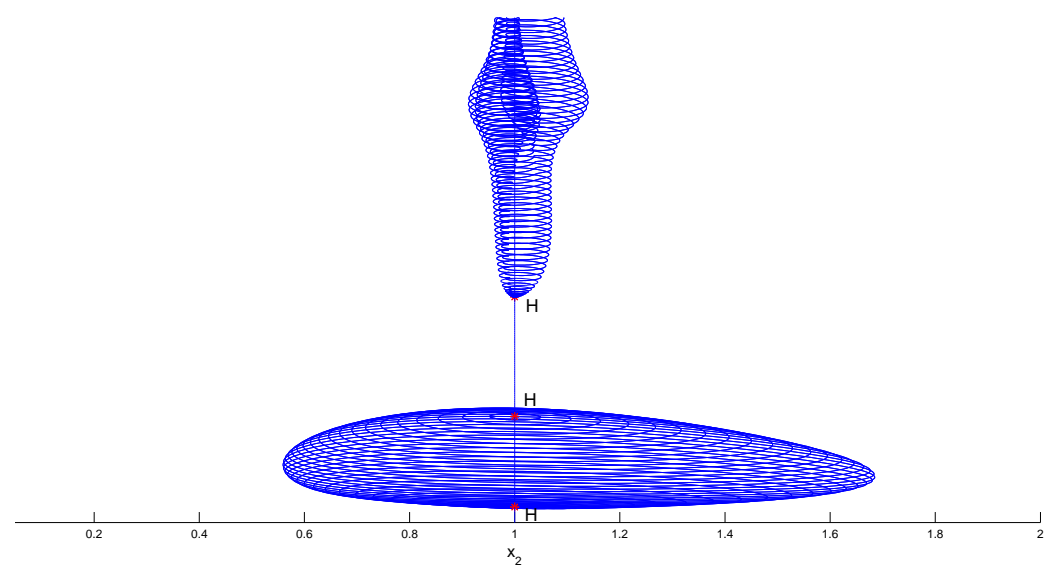

Figure 10: The graph shows a family of stable periodic solutions, where $\mathrm{H}$ represents a bifurcation point. 